\title{
ON SUBGROUPS OF THE MULTIPLICATIVE GROUP OF SKEW FIELDS
}

\author{
A. LICHTMAN
}

\begin{abstract}
We give an example of a skew field $D$ for which the multiplicative group $D^{*}$ of $D$ contains a finitely generated subgroup which satisfies a nontrivial identity and has no solvable normal subgroup of finite index. It gives a negative answer to a question of $\mathrm{S}$. Bachmuth.
\end{abstract}

1. In [1] J. Tits proved that any finitely generated subgroup of a matrix group over a field is either solvable-by-finite or contains a noncyclic free group. At the Second International Conference on the Theory of Groups, S. Bachmuth raised the question of whether Tits' Theorem would remain true if the field were replaced by division ring (see [2, p. 736]).

In this note, we show that Tit's Theorem fails even for matrices of degree one, that is, for division rings themselves. We shall show as a consequence of Theorem 1 below that there exists a finitely generated group which is not solvable-by-finite and does not contain a noncyclic free subgroup, but whose group ring can be embedded in a division ring of quotients.

Let us remark that the question of whether the multiplicative group of any noncommutative division ring contains a noncyclic free subgroup is open.

2. Let $F$ be a noncyclic free group, $N$ a normal subgroup of $F$ and let $V(N) \neq N$ be a fully invariant subgroup of $N$.

We need the following theorem, which was proved in [3].

THEOREM 1. If $\bar{N}=N / V(N)$ is a nilpotent torsion-free group and $G=$ $F / N$ has a subnormal series (possibly of transfinite length),

$$
G \cdots \triangleright H_{\alpha} \cdots \triangleright H_{2} \triangleright H_{1} \triangleright E,
$$

where all factors $H_{\alpha+1} / H_{\alpha}$ are locally finite or locally solvable groups, then the group ring $R(\bar{F})$ of the group $\bar{F}=F / V(N)$ over any field $R$ has no zero divisors and has a division ring of quotients.

(Let us recall that $\bar{F}$ is a torsion-free group (see [5]).)

The negative answer to the above question will be obtained by applying Theorem 1.

THEOREM 2. There exists a division ring $D$ for which the multiplicative group $D^{*}$ of $D$ contains a finitely generated subgroup which satisfies some nontrivial

Received by the editors April 16, 1976.

AMS (MOS) subject classifications (1970). Primary 16A40.

Key words and phrases. Multiplicative group of division ring, group ring. 
identity and has no solvable normal subgroup of finite index.

Proof. Let $P$ be any noncyclic simple finite group. Let us consider the standard wreath product $W=P$ wr $H$, where $H$ is the infinite cyclic group.

The series (1) exists in the group $W$ since $W$ is an extension of the base group $\tilde{P}$, which is locally finite, by the group $H$. We also remark that $W$ is finitely generated.

Now write $W=F / N$, where $F$ is a finitely generated free group and $N \triangleleft$ $F$; for any $k$ let $\Gamma_{k}(N)$ denote the $k$ th member of the lower central series of $N$ and put $\bar{F}=F / \Gamma_{k}(N)$.

Now if $R$ is an arbitrary field, Theorem 1 implies that the group $\operatorname{ring} R(\bar{F})$ has no zero divisors and has a division ring of quotients which we denote by $D$. The group $\bar{F} \subseteq D^{*}$ is finitely generated and is an extension of a free nilpotent group $\bar{N}=N / \Gamma_{k}(N)$ by the group

$$
\bar{F} / \bar{N}=\left(F / \Gamma_{k}(N)\right) /\left(N / \Gamma_{k}(N)\right) \simeq F / N=W .
$$

It follows that $\bar{F}$ satisfies some nontrivial identity.

Let us prove now that $\bar{F}$ has no solvable invariant subgroup of finite index.

Indeed, if $\bar{F}$ is solvable-by-finite, then so is its homomorphic image $W$ and its base subgroup $\tilde{P}$. As $\tilde{P}$ is a direct product of simple groups, any normal subgroup $Q \triangleleft \tilde{P}$ is also a direct product of simple subgroups isomorphic to $P$ (see, for instance, [4, Volume I, Theorem 5.45]). Therefore, $Q$ cannot be solvable.

We have proved that the subgroup $\bar{F}$ satisfies all the demands of Theorem 1.

3. Some further information about the existence of subgroups with identity in the multiplicative group of skew fields is contained in the following theorem.

TheOrem 2'. Let $D$ be a skew field. Then there exists a skew field $D_{1} \supseteq D$ such that its multiplicative group $D_{1}^{*}$ contains a finitely generated subgroup $S$ which satisfies a nontrivial identity and has no solvable subgroup of finite index.

The proof can be obtained by modification of the proofs of Theorems 1 and 2.

I am grateful to the editor and to the referee for useful remarks.

\section{REFERENCES}

1. J. Tits, Free subgroups in linear groups, J. Algebra 20 (1972), 250-270. MR 44 \#105.

2. Proceedings of the Second International Conference on the Theory of Groups, (Canberra, Australia, 1973), Lecture Notes in Math., vol. 372, Springer-Verlag, Berlin and New York, 1974. MR 49 \#9054.

3. A. I. Lichtman, On embedding of group rings in division rings, Israel J. Math. 23 (1976), 288-297.

4. D. J. S. Robinson, Finiteness conditions and generalized soluble groups, Vols. 1, 2, SpringerVerlag, Berlin and New York, 1972. MR 48 \#11314, 11315.

5. G. Baumslag, Wreath products and extensions, Math. Z. 81 (1963), 286-299. MR 27 \# 1503.

Department of Mathematics, Ben-Gurion University of the Negev, BeER-Sheva, Israel 